Subscriber access provided by King Abdullah University of Science and Technology Library

Letter

\title{
Photoresponsive Bridged Silsesquioxane Nanoparticles with Tunable Morphology for Light-Triggered Plasmid DNA Delivery
}

Yevhen Fatieiev, Jonas Croissant, Shahad Alsaiari, Basem

A Moosa, Dalaver H. Anjum, and Niveen M Khashab

ACS Appl. Mater. Interfaces, Just Accepted Manuscript • DOI: 10.1021/acsami.5b07365 • Publication Date (Web): 25 Sep 2015

Downloaded from http://pubs.acs.org on September 28, 2015

\section{Just Accepted}

"Just Accepted" manuscripts have been peer-reviewed and accepted for publication. They are posted online prior to technical editing, formatting for publication and author proofing. The American Chemical Society provides "Just Accepted" as a free service to the research community to expedite the dissemination of scientific material as soon as possible after acceptance. "Just Accepted" manuscripts appear in full in PDF format accompanied by an HTML abstract. "Just Accepted" manuscripts have been fully peer reviewed, but should not be considered the official version of record. They are accessible to all readers and citable by the Digital Object Identifier (DOI®). "Just Accepted" is an optional service offered to authors. Therefore, the "Just Accepted" Web site may not include all articles that will be published in the journal. After a manuscript is technically edited and formatted, it will be removed from the "Just Accepted" Web site and published as an ASAP article. Note that technical editing may introduce minor changes to the manuscript text and/or graphics which could affect content, and all legal disclaimers and ethical guidelines that apply to the journal pertain. ACS cannot be held responsible for errors or consequences arising from the use of information contained in these "Just Accepted" manuscripts. 


\title{
Photoresponsive Bridged Silsesquioxane Nanoparticles with Tunable Morphology for Light-Triggered Plasmid DNA Delivery
}

\author{
Yevhen Fatieiev, ${ }^{\ddagger}$ Jonas G. Croissant, ${ }^{\ddagger}$ Shahad Alsaiari, Basem A. Moosa, Dalaver H. Anjum, and \\ Niveen M. Khashab*
}

Smart Hybrid Materials Laboratory (SHMs), Imaging and Characterization Laboratory, Advanced Membranes and Porous Materials Center, King Abdullah University of Science and Technology (KAUST), Thuwal 23955-690o, Kingdom of Saudi Arabia.

\begin{abstract}
Bridged silsesquioxane nanocomposites with tunable morphologies incorporating o-nitrophenyleneammonium bridges are described. The systematic screening of the sol-gel parameters allowed the material to reach the nanoscale with controlled dense and hollow structures of 100 to $200 \mathrm{~nm}$. The hybrid composition of silsesquioxanes with $50 \%$ of organic content homogenously distributed in the nanomaterials endowed them with photoresponsive properties. Light irradiation was performed to reverse the surface charge of nanoparticles from +46 to $-39 \mathrm{mV}$ via the photoreaction of the organic fragments within the particles, as confirmed by spectroscopic monitorings. Furthermore, such nanoparticles were applied for the first time for the ondemand delivery of plasmid DNA in HeLa cancer cells via light actuation.

KEYWORDS: bridged silsesquioxane, DNA delivery, organosilica, hollow nanoparticles, charge reversal
\end{abstract}

Bridged silsesquioxane (BS) nanomaterials with chemical structures $\mathrm{O}_{1.5} \mathrm{Si}-\mathrm{R}-\mathrm{SiO}_{1.5}$ with organic $\mathrm{R}$ groups are emerging as the next generation of organosilica nanocomposites. ${ }^{1-9}$ Nonetheless, it remains a challenge to control the kinetic in sol-gel processes, which generally lead to macroscaled nonporous BS functional materials. ${ }^{10-12}$ Their design involves kinetically-controlled sol-gel processes of bis- or multiorganoalkoxysilanes which yield to hybrid materials with very high organic contents depending on the type of organic groups. ${ }^{1}$ BS materials substantially differ with organicallymodified silica, also called ORMOSIL, which are organicallydoped silica materials and thus possess lower organic contents. Consequently, the BS matrix photophysical, chemical, thermal and mechanical properties are governed by the homogenously distributed organic fragments within the siloxane network. ${ }^{13}$ Thus, BS materials with features such as luminescence, ${ }^{14}$ magnetism, ${ }^{15}$ and self-assembly were designed ${ }^{16}$ for various applications including catalysis, ${ }^{17}$ solid-state lightning, ${ }^{18}$ energy and electronics. ${ }^{13}$

Nanoscaled BS materials are thus highly desirable in this day of miniaturized devices and nanocomposite materials. Shea et al. pioneered BS nanoparticles (NPs) with photodeformable particles based on coumarin dimer bridges. ${ }^{3}$ The material was composed of $100 \mathrm{~nm}$ aggregated spherical particles. The same group later reported bipyridinium- and ethylenediamine-based BS spherical monodisperse particles, ${ }^{4}$ as well as phenylene, alkylene, and aminoalkyl bridges. ${ }^{19}$ Recently, few studies described BS NPs for biomedical applications, namely drug delivery with cis-platin bridges as prodrug, ${ }^{20}$ MRI imaging ${ }^{21}$ with BS NPs composed of gadoliniumcomplexed bridges. Croissant et al. reported BS and gold core BS shell NPs designed from a tetra-alkoxysilylated twophoton photosensitizer and efficiently applied them for twophoton fluorescence imaging and photodynamic therapy in cancer cells. They recently designed disulfide-based biodegradable BS NPs chemically-doped with diphenylbutadiene and porphyrin photosensitizers for two-photon photodynamic therapy and imaging in-vitro. ${ }^{22}$ BS NPs with charge reversal from negative to positive values were reported with aminopropyl-bridges with o-nitrobenzyl pending groups and applied for light-triggered hydrogel assembly and plastic antibody release. ${ }^{23}$ Reported BS nanomaterials were almost exclusively dense nanospheres, with the exception of perylenediimide-bridged nanoribbons endowed with electronic properties obtained by Hammer and co-workers, and several microfibers with diameters of few hundred nanometres. ${ }^{24}$ The aggregation of BS NPs is often observed and remains a major drawback for future biomedical applications. Ideally, BS NPs should be non-aggregated sub-20o nm nanomaterials to benefit the enhanced permeation and retention (EPR) effect and thus accumulate in cancerous tissues and organs. The delivery of plasmid DNA was achieved with ORMOSIL and mesoporous silica NPs, ${ }^{25-26}$ but not with BS NPs, and necessitated complex multi-step postfunctionalizations of polymer and coupling agent.

Herein we report the controlled syntheses of sub-20o nm non-aggregated BS nanospheres with tunable morphology with dense or hollow nanostructure designed from sol-gel processes of a photoresponsive bridged alkoxysilane (PBA) precursor (see Figure 1). The unique constitution of these BS NPs enables the on-demand surface charge reversal via lighttrigger from positive to negative charges. Furthermore, this feature was harnessed to apply BS nanocarriers without further functionalization for the first time for light-triggered plasmid DNA delivery in cancer cells. 


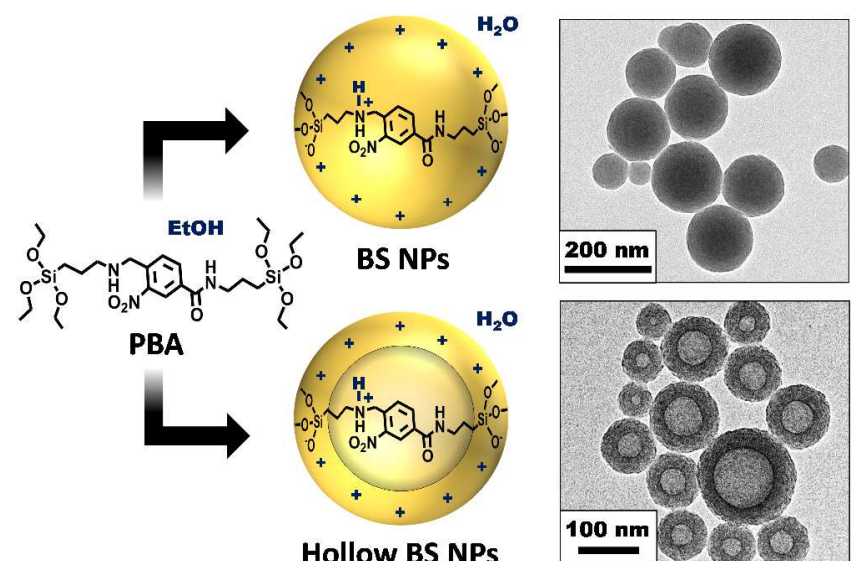

Figure 1. Design of BS and hollow BS NPs via the sol-gel reactions of the PBA precursor, as shown by TEM micrographs.

The PBA precursor, of IUPAC name 3-nitro- $\mathrm{N}-(3-$ (triethoxysilyl)propyl)-4-(((3-(triethoxysilyl)propyl)amino)methyl)benzamide, was first synthesized and characterized. The synthesis involved a soft chlorination of 4(bromomethyl)-3-nitrobenzoic acid followed by a coupling with 2 equivalent of 3 -aminopropyltriethoxysilane in dichloromethane under base-catalysis at $0^{\circ} \mathrm{C}$ (ESI, Figure $\left.\mathrm{S}_{1}\right) .^{27}$ The chemical structure of the precursor was confirmed via proton and carbon nuclear magnetic resonance (NMR) and Fourier transform infrared (FTIR) spectroscopies (Figures S2 and $\mathrm{S}_{3}$ ).

BS nanomaterials were then synthesized via sol-gel methods. Controlled designs of BS NPs with different morphologies were achieved via a modified Stöber method. ${ }^{28}$ An aqueous mixture containing cetyltrimethylammonium bromide (CTAB), sodium hydroxide catalyst, and an ethanol cosolvent was prepared at $75^{\circ} \mathrm{C}$. Then, the hydrolysiscondensation of the PBA precursor was conducted for $2 \mathrm{~h}$. Such conditions lead to the formation of BS NPs with size under $200 \mathrm{~nm}$, as shown by transmission electron microscopy (TEM) image (see top image in Figure 1). Interestingly, we unexpectedly found that without the use of CTAB in a certain range of temperature, $\mathrm{pH}$, and alkoxysilane concentration the formation of the hollow BS nanostructure was observed (see bottom image in Figure 1). In fact, the diameter of NPs as well the formation of the hollow or non-hollow structure could be tuned by varying four parameters: $\mathrm{pH}$, temperature, alkoxysilane concentration and type of alcohol cosolvent (see Figure $\mathrm{S}_{4}$, Tables $\mathrm{S}_{1}$ and $\mathrm{S}_{2}$ ). The formation of hollow particles could be achieved from 50 to $90^{\circ} \mathrm{C}$ and $\mathrm{pH} 11$ to 12 (Figures $\mathrm{S}_{4} \mathrm{~B}-\mathrm{C}$ ), while no correlation was observed between the size and temperature. The possibility to form hollow BS NPs with methanol, ethanol, and i-propanol cosolvent (and not from butanol) suggests the formation of nanoemulsions in specific conditions which promote hollow particle nanostructuration (Figure $\mathrm{S}_{4} \mathrm{D}$ ).

The size, morphology, and composition of BS nanomaterials were further investigated via various techniques. Scanning electron microscopy (SEM) micrographs displayed relatively monodisperse BS and hollow BS spherical NPs (See Figures $\mathrm{S}_{5} \mathrm{~A}-\mathrm{B}$ ), with dynamic light-scattering (DLS) average hydrodynamic diameters of 190 and $106 \mathrm{~nm}$ respectively (Figure $\mathrm{S}_{5} \mathrm{C}-\mathrm{D}$ ), which is in accordance with microscopy images and the hydration layer on NPs. The incorporation of organic bridges into the silica framework of BS and hollow
BS NPs was proved by Fourier transform infrared (FTIR) spectroscopy with the $v_{\mathrm{C}=\mathrm{O}}$ at $1645 \mathrm{~cm}^{-1}, v_{\mathrm{N}-\mathrm{H}}$ at $3289 \mathrm{~cm}^{-1}, v_{\mathrm{N}-\mathrm{O}}$ at $1535 \mathrm{~cm}^{-1}$, as well as aliphatic and aromatic $\mathrm{C}$-H stretching modes (see Figure S6). The high degree of condensation of siloxanes was confirmed by the shift of the $v_{\mathrm{Si}-\mathrm{O}}$ value from 1090 to $1130 \mathrm{~cm}^{-1}$. Thermogravimetric analysis (TGA) of BS and hollow BS NPs also confirmed the high organic content of the nanocomposites with two significant weight losses at 220 and $480^{\circ} \mathrm{C}$ according to the first derivative of the thermogravimetric (DTG) curve, leading to a total weight loss of $48 \%$ (Figures $\mathrm{S}_{7}$ and S8). Moreover, we utilized spectrum imaging (SI) in scanning transmission electron microscopy (STEM) combined with electron energy-loss spectroscopy (EELS) to assess the homogeneity in composition of hollow BS NPs. Spectra were acquired (see one in Figure S9) at each pixel image $(\sim 1 \mathrm{~nm})$ on a representative particle, and the elemental mappings of silicon, oxygen, nitrogen and carbon were extracted (Figure 2). The results clearly exhibit the homogenous dispersion of the organic moieties within the NPs framework. This conclusion was further supported by the homogeneous composition of these elements along the diameter of hollow BS NPs (see Figure Sio). Additionally, we also successfully prepared rhodamine B-doped hollow BS NPs of $50 \mathrm{~nm}$ with homogenous composition of dye in the nanostructure (Figure Si1).

The light-responsiveness of the BS nanomaterials was then assessed and monitored spectroscopically. In a typical experiment,

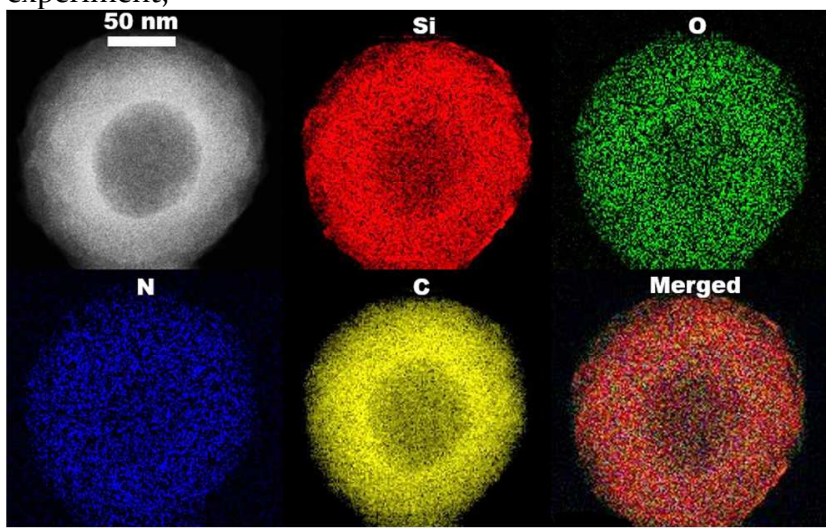

Figure 2. STEM-EELS elemental mapping (silicon, oxygen, nitrogen, carbon) of a representative hollow BS NP. The merged image consists of silicon, nitrogen, and oxygen.

an aqueous suspension of NPs was irradiated with UV-light at $365 \mathrm{~nm}\left(24.6 \mathrm{~mW} . \mathrm{cm}^{-2}\right)$. We first analysed the irradiation of the positively-charged o-nitrophenylene-ammonium PBA precursor which caused a classic internal photocleavage $e^{29-30}$ leading to the formation of the neutral the nitrosophenyleneimine derivative (Figure $3 \mathrm{~A}$ ), as shown by the disappearance of the benzyl protons in the NMR spectrum (see Figure S12). This was supported by the appearance of the shift of the 285 $\mathrm{nm}$ phenyl band in the UV-Visible spectrum (Figure $\mathrm{S}_{13}$ ), and the shift of the symmetric stretching $v_{\mathrm{N}-\mathrm{O}}$ vibration mode from 1540 to $155 \mathrm{o} \mathrm{cm}^{-1}$ (Figure $\mathrm{S}_{14}$ ). However, the same trends were observed in BS nanomaterials (Figure ${ }_{3} \mathrm{~B}-\mathrm{C}$, see FTIR $v_{\mathrm{N}}$ o shift in Figure S15). The photo- reaction monitored via UVVisible spectroscopy was found to necessitate 10 minutes of irradiation for a full conversion (see Figure ${ }_{3} \mathrm{C}$ ), which was accompanied by a progressive modification of the surface charge of BS NPs from +46 to $-5,-30$ and $-39 \mathrm{mV}$ after 4,8 , 
and 10 minutes of irradiation respectively (see Figure $4 \mathrm{~A}$, and Figure S16). Upon illumination, o-nitrophenylene-
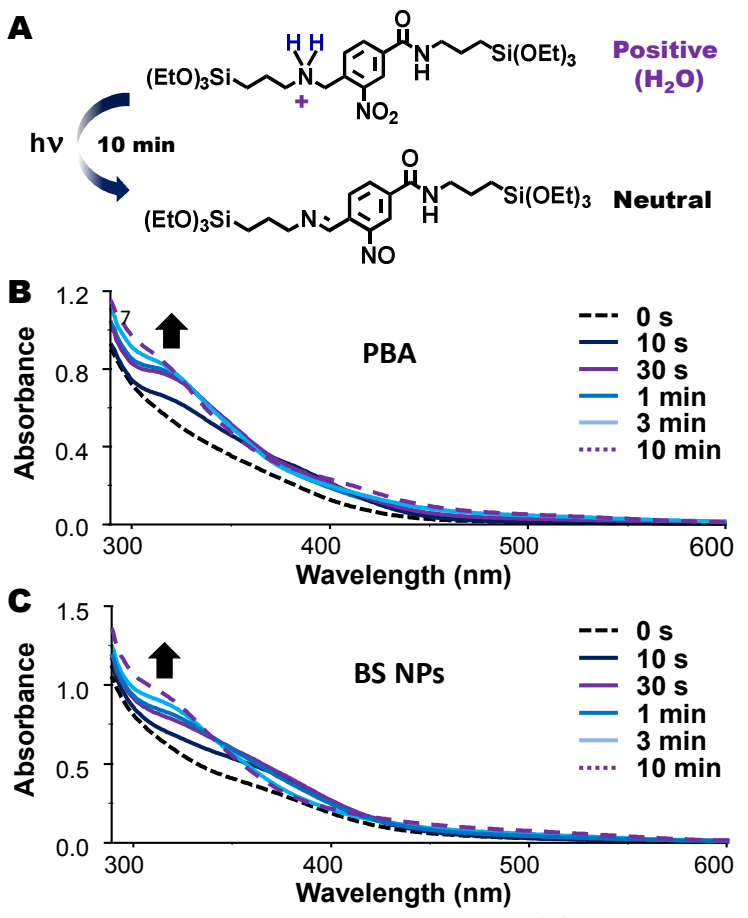

Figure 3. Photoreaction of the PBA precursor (A). UV-Visible spectroscopy monitoring of the photoreaction in the PBA in absolute ethanol (B) and in BS NPs in water (C) before and after irradiation for different time intervals.

$\mathbf{A}$
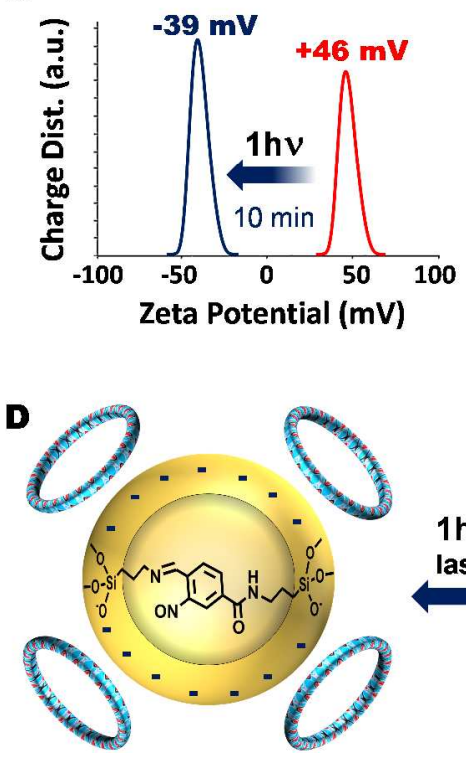

$1 \mathrm{hv}$ laser

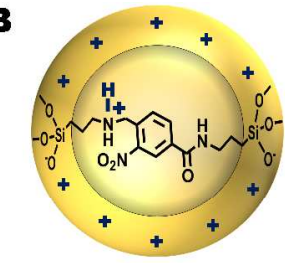

Hollow BS NPS

Figure 4. Zeta potential measurements on BS NPs before and after irradiation, depicting the NPs surface charge reversal (A). Schematic representation of positively-charged BS NPs (B) which electrostatically bind DNA strands $(C)$ for light-triggered delivery (D). The negative charge of NPs results from the neutralisation of the charge of the organic bridges.

ammonium moieties on the surface of BS NPs turned into neutral nitrosophenylene-imine moieties, which resulted in a negative surface charge afforded by silanolates groups (see Figure $\mathrm{S}_{17}$ ).
The unique charge reversal feature was thus applied for the electrostatic binding of negatively charged plasmid DNA and its subsequent delivery to a cancer cell line in culture (Figure $4 \mathrm{~B}-\mathrm{D})$. BS NPs were found to be tolerated by in HeLa cells, as shown by less than $10 \%$ of cytotoxicity up to 20 $\mu \mathrm{g} . \mathrm{mL}^{-1}$ (Figure S18). The electrostatic binding of DNA (6o $\mathrm{pb}, \sim 20 \mathrm{~nm}$ ) on positively charged BS NPs was confirmed via gel electrophoresis and zeta potential measurements, with $7.5 \mathrm{ng}$ of bound DNA per microgram of NPs (Figures S19 and S2o). HeLa cancer cells were then incubated with BS-DNA NPs at $11 \mu \mathrm{g} . \mathrm{mL}^{-1}$ for 12 hours. Nuclei were stained with 4',6diamidino-2-phenylindole (DAPI), and the delivery of plasmid DNAs was assessed via the production of the green fluorescent protein (GFP) as reporter gene. Indeed, the fluorescence of GFP necessitates the transcription of DNA in the nucleus with subsequent mRNA translation, which implies the detachment of DNA from BS NPs given the small pore sizes of nuclei ( $\leq 10 \mathrm{~nm}) .{ }^{26}$ Confocal laser scanning microscopy (CLSM) images were thus acquired to determine the DNA delivery capability of the designed hollow BS NPs (see Figure 5). Before irradiation, a small amount of GFP could be seen, which indicated that few DNA were autonomously delivered to cells via NPs (Figure $5 \mathrm{~A}$ ). However, when the irradiation was turned on $(365 \mathrm{~nm}, 25 \mathrm{~mW}, 4 \times 10 \mathrm{~s})$, a sufficient amount of DNA was delivered in the cytosol (see Figure ${ }_{5} \mathrm{~B}$ ) through photo-induced electrostatic repulsions (Figures ${ }_{4} \mathrm{C}$-D). This effect was also clearly seen after only $6 \mathrm{~h}$ of incubation (Figure S21). Notably, zeta potential measurements for BS NPs didn't show full charge reversal from positive to negative after $40 \mathrm{~s}$ of UV-irradiation. Although, it was enough to decrease electrostatic binding and consequently increase repulsions between negatively charge silanolates groups and DNA. As a result, partial release of DNA from the surface of BSDNA NPs was observed (Figure S20). Note that, single stranded DNA were also attached to BS NPs ( 53 ng. $\mathrm{mg}^{-1}$ ) and transported and tracked via $\mathrm{Cy}_{3}$ cyanine labelling, while bright field images showed the intracellular co-localizations of aggregated BS NPs and DNA-Cy3 fluorescence (Figure S22).

A

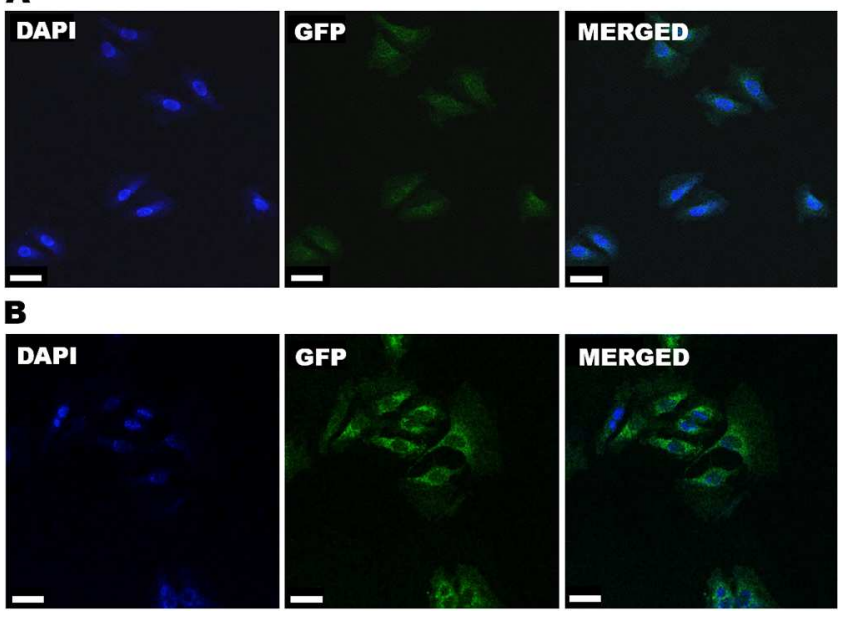

Figure 5. CLSM images on HeLa cells after $12 \mathrm{~h}$ of incubation with non-irradiated (A) and irradiated BS NPs binding DNA strands (B). Nuclei are stained in blue with DAPI. DNA is tracked via GFP fluorescing in green, thus proving the DNA delivery from BS NPs. Scale bars of $40 \mu \mathrm{m}$. 
In summary, we report the syntheses of bridged silsesquioxane nanomaterials with tunable size and morphology, affording non-aggregated dense or hollow nanospheres. The organic-inorganic nanomaterials possessed a very high organic content $(\sim 50 \%)$ of photoresponsive fragments which enabled the on-demand charge reversal from positive to negative values. The hybrid compositions of the designed materials were investigated via various techniques and found to be homogenous in the NPs, while its photoresponsiveness was monitored spectroscopically. By way of proof of principle of the biomedical application of these BS NPs, light-triggered delivery of plasmid DNA to cancer cells in culture was demonstrated for the first time. The lightactuation was found to be effectively delivering DNA while the non-irradiated nanomaterials did not induce significant gene expressions. Dye-doped hollow BS NPs are envisioned for biomedical imaging while the use of a near-infrared fluorophore could extend its potential for in-vivo biomedical applications.

\section{ASSOCIATED CONTENT}

\section{Supporting Information}

Supplementary experimental sections, additional characterization data of the PBA, BS and hollow BS NPs, and data of in vitro studies. This material is available free of charge via the Internet at http://pubs.acs.org.

\section{AUTHOR INFORMATION}

\section{Corresponding Author}

E-mail: Niveen.khashab@kaust.edu.sa Tel: +966-28021172.

Fax: +966-28082410.

\section{Author Contributions}

$\ddagger$ These authors contributed equally. All authors contributed to the work.

Notes

The authors declare no competing financial interests.

\section{ACKNOWLEDGMENTS}

We gratefully acknowledge support from King Abdullah University of Science and Technology (KAUST).

\section{REFERENCES}

(1) Croissant, J.; Maynadier, M.; Mongin, O.; Hugues, V.; Blanchard-Desce, M.; Chaix, A.; Cattoën, X.; Wong Chi Man, M.; Gallud, A.; Gary-Bobo, M.; Garcia, M.; Raehm, L.; Durand, J.-O. Enhanced Two-Photon Fluorescence Imaging and Therapy of Cancer Cells via Gold@Bridged Silsesquioxane Nanoparticles. Small 2015, 11, 295-299.

(2) Xu, L.; Manda, V. R.; McNamara, L. E.; Jahan, M. P.; Rathnayake, H.; Hammer, N. I. Covalent Synthesis of Perylenediimide-Bridged Silsesquioxane Nanoribbons and Their Electronic Properties. RSC Adv. 2014, 4, 30172-30179.

(3) Zhao, L.; Loy, D. A.; Shea, K. J. Photodeformable Spherical Hybrid Nanoparticles. J. Am. Chem. Soc. 2006, 128, 1425014251.

(4) Khiterer, M.; Shea, K. J. Spherical, Monodisperse, Functional Bridged Polysilsesquioxane Nanoparticles. Nano Lett. 2007, 7, 2684-2687.

(5) Croissant, J.; Salles, D.; Maynadier, M.; Mongin, O.; Hugues, V.; Blanchard-Desce, M.; Cattoën, X.; Wong Chi Man, M.; Gallud, A.; Garcia, M.; Gary-Bobo, M.; Raehm, L.; Durand,
J.-O. Mixed Periodic Mesoporous Organosilica Nanoparticles and Core-Shell Systems, Application to in Vitro Two-Photon Imaging, Therapy, and Drug Delivery. Chem. Mater. 2014, 26, 7214-7220.

(6) Croissant, J.; Cattoën, X.; Wong Chi Man, M.; Dieudonné, P.; Charnay, C.; Raehm, L.; Durand, J.-O. One-Pot Construction of Multipodal Hybrid Periodic Mesoporous Organosilica Nanoparticles with Crystal-Like Architectures. Adv. Mater. 2015, 27, 145-149.

(7) Fatieiev, Y.; Croissant, J. G.; Julfakyan, K.; Deng, L.; Anjum, D. H.; Khashab, N. M. Enzymatically Degradable Hybrid Organic-Inorganic Bridged Silsesquioxane Nanoparticles for InVitro Imaging. 2015, 7, 15046-15050.

(8) Blanco, I.; Abate, L.; Bottino, F. A.; Cicala, G.; Latteri, A Dumbbell-Shaped Polyhedral Oligomeric Silsesquioxanes/ Polystyrene Nanocomposites: The Influence of the Bridge Rigidity on the Resistance to Thermal Degradation. J. Compos. Mater. 2015, 49, 2509-2517.

(9) Blanco, I.; Abate, L.; Bottino, F. Synthesis and Thermal Characterization of New Dumbbell-Shaped CyclopentylSubstituted POSSs Linked by Aliphatic and Aromatic Bridges. $J$. Therm. Anal. Calorim. 2015, 121, 1039-1048.

(10) Loy, D. A.; Shea, K. J. Bridged Polysilsesquioxanes. Highly Porous Hybrid Organic-Inorganic Materials. Chem. Rev. 1995, 95, 1431-1442.

(11) Shea, K. J.; Loy, D. A. Bridged polysilsesquioxanes. Molecular-Engineered Hybrid Organic-Inorganic Materials. Chem. Mater. 2001, 13, 3306-3319.

(12) Moreau, J. J.; Pichon, B. P.; Wong Chi Man, M.; Bied, C.; Pritzkow, H.; Bantignies, J. L.; Dieudonné, P.; Sauvajol, J. L. A Better Understanding of the Self-Structuration of Bridged Silsesquioxanes. Angew. Chem., Int. Ed. 2004, 43, 203-206.

(13) Hu, L.-C.; Shea, K. J. Organo-Silica Hybrid Functional Nanomaterials: How Do Organic Bridging Groups and Silsesquioxane Moieties Work Hand-In-Hand? Chem. Soc. Rev. 2011, 40, 688-695.

(14) Nobre, S. S.; Cattoën, X.; Ferreira, R. A.; Carcel, C.; de Zea Bermudez, V.; Wong Chi Man, M.; Carlos, L. D. Eu ${ }^{3+}$-Assisted Short-Range Ordering of Photoluminescent Bridged Silsesquioxanes. Chem. Mater. 2010, 22, 3599-3609.

(15) Wu, L.; Chen, Q.; Lv, Z.; Sun, W.; Chen, L.; Wu, J. Bithiazole-Bridged Polysilsesquioxane and Its Metal Complexes: Synthesis and Magnetic Properties. J. Sol-Gel Sci. Technol. 2011, 60, 214-220.

(16) Creff, G.; Pichon, B. P.; Blanc, C.; Maurin, D.; Sauvajol, J.L.; Carcel, C.; Moreau, J. J. E.; Roy, P.; Bartlett, J. R.; Wong Chi Man, M.; Bantignies, J.-L. Self-Assembly of Bridged Silsesquioxanes: Modulating Structural Evolution via Cooperative Covalent and Noncovalent Interactions. Langmuir 2013, 29, 5581-5588.

(17) Monge-Marcet, A.; Pleixats, R.; Cattoën, X.; Man, M. W. C.; Alonso, D. A.; Nájera, C. Prolinamide Bridged Silsesquioxane as an Efficient, Eco-Compatible and Recyclable Chiral Organocatalyst. New J. Chem. 2011, 35, 2766-2772.

(18) Graffion, J.; Cattoën, X.; Freitas, V. T.; Ferreira, R. A.; Man, M. W. C.; Carlos, L. D. Engineering of Metal-Free Bipyridine-Based Bridged Silsesquioxanes for Sustainable SolidState Lighting. J. Mater. Chem. 2012, 22, 6711-6715.

(19) Hu, L.-C.; Khiterer, M.; Huang, S.-J.; Chan, J. C. C.; Davey, J. R.; Shea, K. J. Uniform, Spherical Bridged Polysilsesquioxane Nano- and Microparticles by a Nonemulsion Method. Chem. Mater. 2010, 22, 5244-5250.

(20) Rocca, J. D.; Werner, M. E.; Kramer, S. A.; HuxfordPhillips, R. C.; Sukumar, R.; Cummings, N. D.; Vivero-Escoto, J. L.; Wang, A. Z.; Lin, W. Polysilsesquioxane Nanoparticles for Triggered Release of Cisplatin and Effective Cancer 
Chemoradiotherapy. Nanomedicine (N. Y., NY, U. S.) 2015, 11, 31-38.

(21) Vivero-Escoto, J. L.; Rieter, W. J.; Lau, H.; HuxfordPhillips, R. C.; Lin, W. Biodegradable Polysilsesquioxane Nanoparticles as Efficient Contrast Agents for Magnetic Resonance Imaging. Small 2013, 9, 3523-3531.

(22) Croissant, J. G.; Mauriello-Jimenez, C.; Cattoën, X.; Wong Chi Man, M.; Raehm, L.; Maynadier, M.; Gary-Bobo, M.; Garcia, M.; Maillard, P.; Durand, J.-O. Synthesis of Disulfide-Based Biodegradable Bridged Silsesquioxane Nanoparticles for TwoPhoton Imaging and Therapy of Cancer Cells. Chem. Commun. 2015, 51, 12324-12327.

(23) Hu, L.-C.; Yonamine, Y.; Lee, S.-H.; van der Veer, W. E.; Shea, K. J. Light-Triggered Charge Reversal of Organic-Silica Hybrid Nanoparticles. J. Am. Chem. Soc. 2012, 134, 1107211075.

(24) Llusar, M.; Sanchez, C. Inorganic and Hybrid Nanofibrous Materials Templated with Organogelators. Chem. Mater. 2008, 20, 782-820.

(25) Roy, I.; Ohulchanskyy, T. Y.; Bharali, D. J.; Pudavar, H. E.; Mistretta, R. A.; Kaur, N.; Prasad, P. N. Optical Tracking Of Organically Modified Silica Nanoparticles as DNA Carriers: a
Nonviral, Nanomedicine Approach for Gene Delivery. Proc. Natl. Acad. Sci. U. S. A. 2005, 102, 279-284.

(26) Torney, F.; Trewyn, B. G.; Lin, V. S. Y.; Wang, K. Mesoporous Silica Nanoparticles Deliver DNA and Chemicals into Plants. Nat. Nanotechnol. 2007, 2, 295-300.

(27) Li, S.; Moosa, B. A.; Croissant, J. G.; Khashab, N. M. Electrostatic Assembly/Disassembly of Nanoscaled Colloidosomes for Light-Triggered Cargo Release. Angew. Chem., Int. Ed. 2015, 127, 6908-6912.

(28) Kresge, C. T.; Leonowicz, M. E.; Roth, W. J.; Vartuli, J. C.; Beck, J. S. Ordered Mesoporous Molecular Sieves Synthesized By Liquid-Crystal Template Mechanism. Nature 1992, 359, 710 712.

(29) Il'ichev, Y. V.; Schwörer, M. A.; Wirz, J. Photochemical Reaction Mechanisms of 2-Nitrobenzyl Compounds: Methyl Ethers and Caged ATP. J. Am. Chem. Soc. 2004, 126, 4581-4595.

(30) Smet, M.; Liao, L.-X.; Dehaen, W.; McGrath, D. V. Photolabile Dendrimers Using o-Nitrobenzyl Ether Linkages. Org. Lett. 2000, 2, 511-513. 


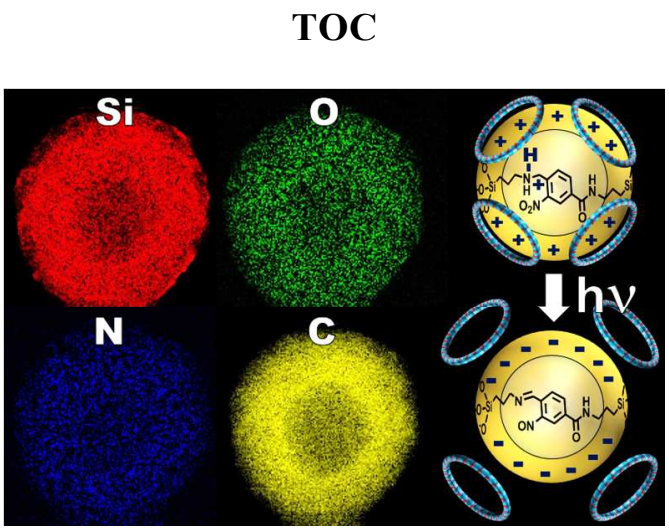

